of stitch which I experimented with. In working this out on inanimate objects it answered perfectly well, but when it came to applying it to the living subject it was found that during the process of healing in the wound the chain got, as it were, fouled by the uniting tissue and the stitch would not pull out easily. Being dissatisfied with this I then tried the other form of sewing-machine or "lock" stitch, and this was found quite satisfactory and perfectly easy of removal provided certain points in its application were attended to.

In the ordinary sewing-machine the efficiency and neatness of the stitching depend upon preserving the ratio between the tensions of the two threads employed. If the tension is properly regulated the interlocking of the threads will take place in the substance of the material under suture, whereas if the tension of one of the threads be greater than that of the other the interlocking of the threads will take place on the surface of the material and on the side of the thread with the highest tensic $n$. By using two threads with different pliability in surgical suturing the same effect can be produced as would be caused by extreme difference in the tension in machining; thus, if one thread be of silk and the other of, say, stiff steel wire it is obvious that instead of the threads interlocking in the substance of the material they will hardly interlock at all, but the more pliable silk will simply pass round the stiff wire and the turns will all be on the side of the material to which the wire is applied, and, further, it will be found that on pulling the wire this can be easily withdrawn and in consequence the silk, having lost its support, can also be withdrawn with the slightest pull.

The method of introducing the suture is of the simplest. All that is required is a mounted needle with an eye near the point; through this is threaded a long piece of silk. This forms the primary thread; the secondary thread is best formed by a stout piece of silkworm-gut which is sufficiently rigid to avoid kinking and being drawn into the sutured tissue. Supposing the peritoneum is to be sutured after a laparotomy the procedure will be as follows:- The two layers of the peritoneum which are to be sewn together are defined the mounted needle, threaded, with the long end of the thread on the left hand or upper side of the ntedle when held as it will be when piercing the tissue, is passed from the operator through both layers of the peritoneum, beginning at the lower angle of the wound. The needle is then withdrawn, leaving a loop of silk protruding through the layers of peritoneum on the side of the assistant; through this loop the assistant passes the end of the silkworm-gut, from below upwards, and the loop is withdrawn flush with the assistant's side of the united flaps. The needle, not having been unthreaded, is again thrust through the flaps for the second stitch and again withdrawn, leaving a loop as in the first instance; the silkworm-gut is threaded through this second loop and the process is repeated till the whole of the flaps are united these being held together by a series of silk loops, the primary thread, held in position by the secondary thread of silkworm-gut passing through the loops on the assistant's side. The needle is next unthreaded, leaving a fairly long end to the silk. There will be now on the face of the wound, on the side of the surgeon, the two ends of silk, one entering the peritoneal flaps at the lower end of the wound and the other leaving the peritoneum at the upper end, and on the side of the assistant the silkworm-gut thread passing through the silk loops and also with long ends, one at the lower end of the wound and the other at the npper end. The silkworm-gut thread should now be put on the stretch and pulled gently backwards and forwards to see that it is nowhere kinked and that it can be withdrawn. This having been done, the four ends, two silk and two silkworm-gut, are each in turn threaded on an ordinary needle and made to pierce the skin on its own side of the wound and are left thus whilst the wound in the abdominal wall is sutured in the ordinary way. This having been done the two lower ends of the peritoneal suture, being one of silk and the other of silkworm-gut, are then tied together and cut to a convenient length. The same is done with the two ends at the upper end of the wound and the process is complete.

When it is required to remove the deep stitch, which may be any time after the wound is healed, all that is required is to cut the lower united stitches on the silk side of the knot and the upper stitch on the gut side of the knot, or vice vers $\hat{a}$, and to pull out first the silkworm.gut stitch which will offer no resistance, and then the silk stitch which having lost its support can be pulled out with equal facility.
The suturing itself can be done quite as rapidly as the ordinary continued suture and the only point requiring special attention is not to pull the silk loops so tight that they drag the silkworm-gut thread into the stitch hole or on to the silk side of the wound.

The method is applicable to any form of buried suture which it may be desirable to remove after healing, except that if the suturing has to take a curve of more than about a sextant two or more separate silkworm gut threads will have to be used in following the curve; otherwice, if the one thread has to curve round too great a part of a circle it will be found to drag on the loops on an attempt at withdrawal and will not run.

There would appear to be no limit, in reason, to the time that the suture may be left in position without causing trouble, provided the part be kept aseptic; thus, in cases of radical cure of hernia I bave kept the stitches closing the ring in position for 15 days without any sign of suppuration or irritation and with no indication that such was likely to occur, and at the end of that time have removed the stitches without the slightest difficulty.

Up to the present I have employed the stitch in cases of laparotomy, in radical cure of hernia, in suprapubic cystotomy, for suturing the pleura in cases of abscess of the liver, and in colporrhaphy, and have found it to answer very well

Cairo.

\section{A CASE OF TYPHOID PANCREATITIS.}

By B. G. A. MOYNIHAN, M.S. LOND., F.R.C.S. ENG., SENIOR ASSISTANT SURGEON TO THE LEEDS GENERAL INFIRMARY; CONSULTING SURGEON TO THE SKIPTON HOSPITAL AND TO THE MIRFIELD MEMORIAL HOSPITAL.

Ix has been generally recognised, since Riedel in 1892 first drew attention to the fact, that chronic pancreatitis is not infrequently dependent upon the irritation of gall-stone disease. The constantly recurring attacks of cholangitis aroused by the passing of a stone down the cystic and common ducts, the impaction of a stone in the lower end of the common duct, and especially its lodgment in the ampulla of Vater, may all be the determining causes of a chronic inflammation of the pancreas.

Among causes other than gall-stones which are capable of originating an acute inflammation of any part of the bile passages typhoid fever, as shown by Murchison, Keen, and many others, takes a prominent place. The bacillus typhosus may be found in the bile for many months or even years after an attack of typhoid fever. In one case a pure culture of the organism was obtained from the bile in the gall-bladder after seven years. Since the common bile-duct and the duct of the pancreas have a common opening into the duodenum at the ampulla of Vater it is not difficult to understand how an ascending infection attacking the bile passages may also affect the canal of Wirsung. In the following case there was a condition of typhoid pancreatitis associated with an infection of the gall-bladder by the bacillus typhosus.

The patient, a boy, aged 13 years, who was s $\epsilon$ nt to me by Dr. W. A. H. Waite of Leeds, was admitted to the Leeds General Infirmary on March 2nd, 1903. The history of the case was that he had been in his usual good health up to September, 1901. In that month he had typhoid fever, from which he made a slow but satisfactory recovery. At frequent intervals since his convalescence he complained of attacks of pain in the upper part of the abdomen. When asked to point out the seat of the most severe pain he laid his hand across the epigastrium. The pain on several occasions had lasted for three or four hours and had been accompanied by feelings of nausea and, though rarely, by vomiting. In November, 1902, for the first time such an attack was followed by jaundice which lasted for several days and then gradually cleared away. Almost every week since November similar attacks of pain, varying in intensity, had occurred and the jaundice had deepened slightly after each attack. The jaundice had never cleared away completely, though it had varied much in depth of tinge. There had never been any elevation of temperature when the pain had come and there had been neither shivering nor flushing. For the last three months the appetite had been poor and the boy had become considerably emaciated. 
On examination of the abdomen there was distinct tenderness in all the region above the level of the umbilicus. Nothing abnormal could be felt. A diagnosis of typhoid infection of the gall-bladder, probably resulting in gallstones one of which had stayed in the common duct, was made. On March 6th the abdomen was opened. The gallbladder contained bile but no stones. There were no adhesions around the bladder or the bile-ducts. There was no stone in any part of the bile passages. The cause of the jaundice was found in the pancreas. The head and much of the body of the pancreas were found to be at least $t$ wice as large as the normal and almost as hard as stone. I have never felt even in old-standing cases of chronic indurating pancreatitis a gland so intensely hard as this was. The common duct was obviously buried in a mass of densely hard inflammatory material in the head of the pancreas. The gall-bladder was aspirated and about half an ounce of bile was withdrawn into a sterilised vessel. A drainage-tube was sutured into the gall-bladder and the abdominal wound was closed in the usual way. The bile was handed over to Dr. J. A. Cairns Forsyth for examination. His report read as follows:-

An agar tube was inoculated with the bile. The resulting growth was examined and found to be composed of bacilli resembling the bacillus coli or the bacillus typhosus. The bacilli had the following properties: (1) in fluid gelatin they cause a uniform turbidity withou the formation of any flocculi ; (2) they do not coagulate or curdle milk and (3) they do not produce an acid reaction on litmus agar. The organisms are therefore typhoid bacilli.

Dr. Forsyth then examined the blood for Widal's reaction and reported as follows:-

The serum gave a positive reaction-1 in 20 instantaneously, 1 in 60 in ten minutes, and 1 in 100 in 40 minutes.

The gall-bladder was drained for four weeks. Recovery was uninterrupted and in all respects most satisfactory.

This case is, so far as I can ascerlain, the first in which an inflammation of the pancreas due to typhoid fever has been recognised. I have operated now upon 13 cases of chronic pancreatitis, but in no instance have $I$ observed so gross an alteration in the size and consistence of the gland. At the end of three weeks the bile discharyed from the gall-bladder was examined by Dr. Forsyth and was found to contain very large numbers of typhoid bacilli. I then ordered urotropine in ten-grain doses thrice daily, the dose being increased to 15 grains four days later. On the eleventh day after the commencement of the giving of the drug the bile was again examined and was found to contain no typhoid organisms, only "a few cocci."

Leeds.

\section{SOME OBSERVATIONS ON MOVEABLE KIDNEY. ${ }^{1}$}

By T. E. GORDON, M. B. Dub., F.R C.S. IREL., SURGEON To The adexaIDE hospital, DUBLIN.

I WISH in this communication to draw attention to some points of interest relative to moveable kidney and to show the conclusions to which I bave come. I have seen very many cases of this condition and $I$ have notes of 12 cases in which I performed a fixation operation. Of these 12 cases all the patients were women except one who was a young man. In all of these cases I operated by the same methodi.e., that recommended by Morris-with one modification. In all the result was aseptic. I do not think permanent complete fixation has been the rule, but, as Edebohls has pointed out, a certain degree of return of mobility is not inconsistent with perfect $s$ uccess as regards the relief of symptoms.

It is now well recognised that moveable kidney is a cause of various and diverse symptoms-that it may induce vomiting and other gastric manifestations, jaundice and hepatic colic, or symptoms which be'ong to the kidney itself, and that it may be associated with a neurasthenic condition. With such diversity of symptoms it can readily be understood that moveable kidney is a common cause of mistaken diagnosis. Sometimes cases of moveable kidney are mistaken for something else-e.g., those in which vomiting occurs are supposed to be cases of gastric ulcer or those with jaundice to be cases of gall-stones. On the other hand, symptoms due

I A paper rea 3 before the Dublin Biołogical Club on March 24th, 1903. to something else may be supposed to be due to moveable kidney and this kind of mistake is apt to be attended with serious consequences, as in a case to which I must refer in some detail presently. There are two facts which when fully realised ought to safeguard one against both classes of error to some extent. First it shouid be remembered that moveable kidney is of exceedingly common occurrence and often is the sole cause of some of those symptoms to which I have alluded. In the second place, it should still more carefully be borne in mind that the moveable kidney, whatever degree of mobility it may possess, may be a cause of no symptoms.

We may conveniently arrange these cases of moveable kidney which cause symptoms into certain groups as follows : (1) those with slight discomfort, such as a dragging sensation and associated with vague general ill-health, with or without hysterical manifestations; (2) those in which the symptoms are of the gastro-intestinal type; (3) those with hepatic symptoms; and (4) those having distinctly renal symptoms. I think that the last must be very uncommon. At all events, I have not met with a single case and I will not therefore allude to them further.

Those cases of the first group with hysterical symptoms present special diffisulties. Hysterical women often have a moveable kidney; they are often unaware of this and have no symptoms referable to it. In these circumstances it is obviously a folly to advise nephropexy or, indeed, to make the patient aware that her kidney is moveable. If, however, she is made aware one way or another that she has a moveable kidney an operation may do good. I have had one remarkable success in the case of a woman of this class.

CASE 1.- The patient was emaciated, complained of pain and fulness after eating, was much constipated, and was quite unfit for work. She was Dr. H. T. Bewley's patient and having fruitlessly tried ordinary remedies he handed her over to my care. I fixed her kidney in January, 1901, kept her in bed for six weeks, and had her massaged during part of this time and well fed. 1 saw her again about six months after the operation and found her in perfect health, scarcely to be recognised as her face had so filled out, and able to perform her day's work without discomfort. She had put on three stones in weight. I can justly claim this as a success, but in so doing I must confess to a failure in another case of the same class.

I have now something to say about the more interesting and satisfactory group presenting gastric or gastro-intestinal symptoms. I may begin by recording a fortunate case as I must end by telling of one which was most unfortunate.

CASE 2.-The patient was a young woman who had been treated for several years for a supposed gastric ulcer. She had suffered from repeated attacks of vomiting and pain. The pain was at first felt in her back in the lumbar region but for the last two or three years in the right hypochondrium. An attack might last with remissions from a few days to a month and then she might have an interval of perfect health for several months. It is specially noted by Dr. G. Peacocke, under whose care she was in the hospital, that the pains and vomiting were quite independent of food, and, further, it was stated that an attack never commenced while she was in bed and that it was sometimes relieved by lying down. She had on no occasion vomited blood. Dr. Peacocke found her right kidney very moveable and at his request I performed a fixation operation. This was in October, 1901, and since then the patient has been in perfect comfort and has only vomited once.

This case is an instance of the very common mistake of assigning to organic disease of the stomach symptoms that are due to moveable kidney. The differential diagnosis is, I think, sufficiently brought out in the details which I have given. As an example of the opposite kind of mistake I may just mention a case I saw a year or two ago in which, with stomach symptoms, there was felt a tumour in the left hypochondrium which descended from under the ribs on aspiration and closely simulated moveable kidney.

CASE 3 - The case was Dr. F. T. Heuston's and an exploratory laparotomy showed the tumour, which I thought was a moveable kidney, to be a massive cancer of the stomach which had been dragged by its weight into an unusual position. As comment, I may point out that the kidney-like tumour was on the left side, that the right kidney was not moveable, and that a left kidney is rarely if ever moveable without its fellow on the right side being moveable also.

Moveable right kidney may be a cause not only of pain and vomiting but also of an actual dilatation of the stomach 